PROCEEDINGS OF THE AMERICAN MATHEMATICAL SOCIETY

Volume 135, Number 4, April 2007, Pages 1141-1144

S 0002-9939(06)08681-3

Article electronically published on October 11, 2006

\title{
A SCHOTTKY-TYPE THEOREM FOR STARLIKE DOMAINS IN BANACH SPACES
}

\author{
JORGE MUJICA AND PAULA TAKATSUKA
}

(Communicated by Jonathan M. Borwein)

\begin{abstract}
We show that if $U$ is a starlike domain in a Banach space $E$ and $\mathcal{F}$ is a family of holomorphic functions on $U$ that omit two distinct values and is bounded at the origin, then $\mathcal{F}$ is uniformly bounded on each $U$-bounded set.
\end{abstract}

\section{INTRODUCTION}

Let $E$ be a complex Banach space and let $\mathcal{H}(U)$ denote the vector space of all holomorphic functions on an open subset $U$ of $E$.

Let $\Delta$ denote the open unit disc. The classical Schottky Theorem asserts that for each $0<\alpha<\infty$ and $0<\beta<1$, there is a constant $c(\alpha, \beta)>0$ such that, if $f \in \mathcal{H}(\Delta)$ is a function that omits the values 0 and 1 and satisfies $|f(0)| \leq \alpha$, then $|f(z)| \leq c(\alpha, \beta)$ for every $z \in \beta \Delta$. See Montel (4, p. 86) or Carathéodory ([1], p. 201).

In a recent paper Paula Takatsuka used the classical Schottky Theorem to prove that, for each $0<\alpha<\infty$ and $0<\beta<1$, there is a constant $c(\alpha, \beta)>0$ such that, given a Banach space $E$ (or even a locally convex space $E$ ), a point $x_{0} \in E$ and a balanced open set $U \subset E$, if $f \in \mathcal{H}\left(x_{0}+U\right)$ is a function that omits the values 0 and 1 and satisfies $\left|f\left(x_{0}\right)\right| \leq \alpha$, then $|f(x)| \leq c(\alpha, \beta)$ for every $x \in x_{0}+\beta U$. See Takatsuka ([6], Corollary 15).

In Section 2 of this paper we obtain a Schottky-type theorem for starlike domains in Banach spaces. More precisely, we prove that if $U \subset E$ is a starlike domain and $\mathcal{F} \subset \mathcal{H}(U)$ is a family of holomorphic functions that omit the values 0 and 1 and is bounded at the origin, then $\mathcal{F}$ is uniformly bounded on each $U$-bounded set. In particular, $\mathcal{F}$ is contained and bounded in $\mathcal{H}_{b}(U)$, the space of all holomorphic functions of bounded type on $U$.

In Section 3 we obtain a Schottky-type theorem for arbitrary domains in Banach spaces. More precisely, we prove that if $U \subset E$ is a connected open set and $\mathcal{F} \subset \mathcal{H}(U)$ is a family of holomorphic functions that omit the values 0 and 1 and is bounded at some point, then $\mathcal{F}$ is uniformly bounded on each ball which lies strictly inside $U$. In particular, $\mathcal{F}$ is contained and bounded in $\mathcal{H}_{d}(U)$, the space of holomorphic functions recently studied by Dineen and Venkova [3].

Received by the editors November 11, 2005.

2000 Mathematics Subject Classification. Primary 46G20, 46E50.

Key words and phrases. Banach space, balanced domain, starlike domain, holomorphic function, holomorphic function of bounded type.

The second author was supported by a teaching assistantship from UNICAMP.

(C)2006 American Mathematical Society Reverts to public domain 28 years from publication 
We refer to the books of Dineen [2] or Mujica [5] for background information on infinite-dimensional complex analysis.

\section{A SchottKy-Type theorem in STARLIKe Domains}

A set $U \subset E$ is said to be starlike if $\lambda x \in U$ for all $x \in U$ and $\lambda \in[0,1] . U$ is said to be $x_{0}$-starlike if the set $U-x_{0}$ is starlike, that is, $(1-\lambda) x_{0}+\lambda x \in U$ for all $x \in U$ and $\lambda \in[0,1]$. If $U$ is $x_{0}$-balanced, then $U$ is $x_{0}$-starlike. If $U$ is convex, then $U$ is $x_{0}$-starlike for each $x_{0} \in U$.

If $U \subset E$ is open and $x \in U$, let $d_{U}(x)$ denote the distance from $x \in U$ to the boundary of $U$, that is:

$$
d_{U}(x)=\sup \{r>0: B(x, r) \subset U\} .
$$

Theorem 1. Let $U$ be a starlike open subset of a Banach space and let

$$
U_{n}:=\left\{x \in U:\|x\|<n \text { and } d_{U}(x)>1 / n\right\}
$$

for every $n \in \mathbb{N}$. Then for each $0<\alpha<\infty$, there is a sequence $\left\{c_{n}(\alpha)\right\}_{n=1}^{\infty}$ of positive constants such that, given a function $f \in \mathcal{H}(U)$ that omits the values 0 and 1 and satisfies $|f(0)| \leq \alpha$, then

$$
|f(x)|<c_{n}(\alpha) \text { for all } x \in U_{n} \text { and } n \in \mathbb{N} .
$$

Proof. For each $n$ let $V_{n}:=\operatorname{st}\left(U_{n}\right)=\left\{\lambda x: x \in U_{n}, \lambda \in[0,1]\right\}$. Then $U_{n} \subset V_{n} \subset U$ and each $V_{n}$ is a starlike open set.

Let $n_{0}$ be the first $n \in \mathbb{N}$ such that $0 \in U_{n}$. Fix $n \geq n_{0}$ and let $\varepsilon:=\frac{1}{4 n^{3}}$. We will show that

$$
B(y, 2 \varepsilon) \subset U \text { for every } y \in V_{n} .
$$

Indeed let $y=\lambda x$, with $x \in U_{n}$ and $\lambda \in[0,1]$. We distinguish two cases:

(i) Assume $0 \leq \lambda \leq \frac{1}{2 n^{2}}$. Then, since $\|x\| \leq n$, it follows that $\|\lambda x\| \leq \frac{1}{2 n}$, and therefore

$$
B(\lambda x, 2 \varepsilon)=B\left(\lambda x, \frac{1}{2 n^{3}}\right) \subset B\left(\lambda x, \frac{1}{2 n}\right) \subset B\left(0, \frac{1}{n}\right) \subset U .
$$

(ii) Assume $\frac{1}{2 n^{2}} \leq \lambda \leq 1$. Then, since $B\left(x, \frac{1}{n}\right) \subset U$, it follows that

$$
U \supset \lambda B\left(x, \frac{1}{n}\right)=B\left(\lambda x, \frac{\lambda}{n}\right) \supset B\left(\lambda x, \frac{1}{2 n^{3}}\right)=B(\lambda x, 2 \varepsilon) .
$$

This proves (11). Note also that $V_{n} \subset B(0, m \varepsilon)$, where $m=4 n^{4}$.

Let $f$ and $\alpha$ be as in the statement of the theorem. We will show the existence of constants $\alpha \leq \alpha_{1} \leq \alpha_{2} \leq \cdots \leq \alpha_{m}$ such that

$$
|f(x)| \leq \alpha_{k} \text { for all } x \in V_{n} \cap B(0, k \varepsilon), \quad 1 \leq k \leq m .
$$

Indeed, since $0 \in V_{n}, B(0,2 \varepsilon) \subset U$ and $|f(0)| \leq \alpha$, an application of [6], Corollary 15 , yields a constant $\alpha_{1}:=c\left(\alpha, \frac{1}{2}\right) \geq \alpha$ such that

$$
|f(x)| \leq \alpha_{1} \text { for all } x \in B(0, \varepsilon) .
$$

Now assume that the inequality (2) holds for some $k$, with $1 \leq k \leq m-1$. Let $x \in V_{n} \cap B(0,(k+1) \varepsilon)$, and let $y:=\frac{k}{k+1} x$. Then $y \in V_{n} \cap B(0, k \varepsilon)$ and $x \in B(y, \varepsilon)$. Since $B(y, 2 \varepsilon) \subset U$ and $|f(y)| \leq \alpha_{k}$, an application of [6], Corollary 15, yields a constant $\alpha_{k+1} \geq \alpha_{k}$ such that $|f(x)| \leq \alpha_{k+1}$. This proves (2).

Since $U_{n} \subset V_{n} \subset B(0, m \varepsilon)$, it follows that

$$
|f(x)| \leq \alpha_{m} \text { for all } x \in U_{n} .
$$


Thus we may take $c_{n}(\alpha):=\alpha_{m}$ if $n \geq n_{0}$. If $n<n_{0}$, then $U_{n} \subset U_{n_{0}}$ and we may take $c_{n}(\alpha):=c_{n_{0}}(\alpha)$. This completes the proof.

Corollary 2. Let $U$ be an $x_{0}$-starlike open subset of a Banach space and let

$$
U_{n}:=\left\{x \in U:\left\|x-x_{0}\right\|<n \text { and } d_{U}(x)>1 / n\right\}
$$

for every $n \in \mathbb{N}$. Then for each $0<\alpha<\infty$, there is a sequence $\left\{c_{n}(\alpha)\right\}_{n=1}^{\infty}$ of positive constants such that, given a function $f \in \mathcal{H}(U)$ that omits the values 0 and 1 and satisfies $\left|f\left(x_{0}\right)\right| \leq \alpha$, then

$$
|f(x)|<c_{n}(\alpha) \text { for all } x \in U_{n} \text { and } n \in \mathbb{N} \text {. }
$$

A set $A \subset U$ is said to be $U$-bounded if $A$ is bounded in $E$ and there exists $\varepsilon>0$ such that $A+B(0, \varepsilon) \subset U$. Each $U_{n}$ is $U$-bounded and each $U$-bounded set is contained in some $U_{n}$. $\mathcal{H}_{b}(U)$ denotes the space of all $f \in \mathcal{H}(U)$ which are bounded on all $U$-bounded sets. We endow $\mathcal{H}_{b}(U)$ with the topology of uniform convergence on all $U$-bounded sets. Then we easily get the following corollary.

Corollary 3. Let $U$ be an $x_{0}$-starlike open subset of a Banach space and let $\mathcal{F} \subset$ $\mathcal{H}(U)$ be a family of functions that omit two distinct values. If the family $\mathcal{F}$ is bounded at the point $x_{0} \in U$, then $\mathcal{F}$ is a bounded subset of $\mathcal{H}_{b}(U)$.

\section{A SChOTTKY-TYPE THEOREM IN ARBITRARY DOMAINS}

Theorem 4. Let $U$ be a connected open subset of a Banach space and let $x_{0} \in U$. Let $0<\alpha<\infty$. Then for each $a \in U$ and $0<r<d_{U}(a)$, there is a constant $c(a, r, \alpha)>0$ such that, given a function $f \in \mathcal{H}(U)$ that omits the values 0 and 1 and satisfies $\left|f\left(x_{0}\right)\right| \leq \alpha$, then

$$
|f(x)| \leq c(a, r, \alpha) \text { for all } x \in B(a, r) .
$$

Proof. If $a$ is a point of $U$, let $\gamma$ be a path in $U$ from $x_{0}$ to $a$. Since the trace of $\gamma$ is compact and connected, we can find a finite number of balls $B\left(x_{0}, r_{0}\right), B\left(x_{1}, r_{1}\right), \ldots$, $B\left(x_{n}, r_{n}\right)=B\left(a, d_{U}(a)\right)$ in $U$ with centers on the trace of $\gamma$ such that $x_{k+1}$ and $x_{k}$ are in $B_{k+1} \cap B_{k}$ and $B\left(x_{k}, 2 r_{k}\right) \subset U$ for $0 \leq k \leq n-1$.

Applying [6], Corollary 15, on the ball $B\left(x_{0}, 2 r_{0}\right)$ we obtain a constant $\alpha_{0}:=$ $c\left(\alpha, \frac{1}{2}\right)$ such that

$$
|f(x)| \leq \alpha_{0} \text { for all } x \in B\left(x_{0}, r_{0}\right) .
$$

A second application of [6], Corollary 15, yields another constant $\alpha_{1}:=c\left(\alpha_{0}, \frac{1}{2}\right)$ such that

$$
|f(x)| \leq \alpha_{1} \text { for all } x \in B\left(x_{1}, r_{1}\right) .
$$

Continuing this way, for the last step of this inductive process we first notice that each $0<r<d_{U}(a)$ is of the form $r=\beta d_{U}(a)$, with $0<\beta<1$, and since we have $|f(a)| \leq \alpha_{n-1}$, [6], Corollary 15, provides the constant $c(a, r, \alpha):=c\left(\alpha_{n-1}, \beta\right)$ such that

$$
|f(x)| \leq c(a, r, \alpha) \text { for all } x \in B(a, r) .
$$

Following [3] we denote by $\mathcal{H}_{d}(U)$ the space of all $f \in \mathcal{H}(U)$ which are bounded on all balls $B(a, r)$, with $a \in U$ and $0<r<d_{U}(a)$. We endow $\mathcal{H}_{d}(U)$ with the topology of uniform convergence on all such balls. Clearly $\mathcal{H}_{b}(U) \subset \mathcal{H}_{d}(U) \subset \mathcal{H}(U)$, and Dineen and Venkova 3 have given examples where $\mathcal{H}_{b}(U)=\mathcal{H}_{d}(U)$ and where $\mathcal{H}_{b}(U) \neq \mathcal{H}_{d}(U)$. We then get the following corollary. 
Corollary 5. Let $U$ be a connected open subset of a Banach space and let $\mathcal{F} \subset \mathcal{H}(U)$ be a family of functions that omit two distinct values. If the family $\mathcal{F}$ is bounded at some point $x_{0} \in U$, then $\mathcal{F}$ is a bounded subset of $\mathcal{H}_{d}(U)$.

\section{Concluding Remarks}

If $E$ is finite dimensional, then $\mathcal{H}_{b}(U)=\mathcal{H}_{d}(U)$ and the conclusions of Corollary 3 and Corollary 5 coincide. But if $E$ is infinite dimensional, then $\mathcal{H}_{b}(U) \neq \mathcal{H}_{d}(U)$ in general, as Dineen and Venkova [3] have shown, and thus the conclusion of Corollary 3 is strictly stronger than the conclusion of Corollary 5. It would be important to extend the validity of Corollary 3 to other domains, for instance to polynomially convex domains.

\section{ACKNOWLEDGEMENTS}

This paper is based on part of Paula Takatsuka's doctoral dissertation at UNICAMP, supervised by Jorge Mujica.

We are indebted to Daniela Vieira for calling our attention to the paper of Dineen and Venkova 3 .

\section{REFERENCES}

[1] Carathéodony, C., Theory of Functions of a Complex Variable, Vol. II, Chelsea, New York, 1960.

[2] Dineen, S., Complex Analysis on Infinite-Dimensional Spaces, Springer-Verlag, London, 1999. MR.1705327 (2001a:46043)

[3] Dineen, S. And Venkova, M., Extending bounded type holomorphic mappings on a Banach space, J. Math. Anal. Appl. 297 (2004), 645-658. MR2088686 (2005j:46027)

[4] Montel, P., Leçons sur les Familles Normales de Fonctions Analytiques et leurs Applications, Chelsea, New York, 1974.

[5] Mujica, J., Complex Analysis in Banach Spaces, North-Holland Mathematics Studies 120, North-Holland, Amsterdam, 1986. MR0842435 (88d:46084)

[6] Takatsuka, P., Normal families of holomorphic functions on infinite dimensional spaces, Portugal. Math., to appear.

IMECC - UNICAMP, Caixa Postal 6065, 13083-970 Campinas, SP, Brazil

E-mail address: mujica@ime.unicamp.br

IMECC - UNICAMP, Caixa Postal 6065, 13083-970 Campinas, SP, Brazil

E-mail address: ptakatsu@ime.unicamp.br 